\title{
EL ARBITRAJE EN UN NUEVO SISTEMA INTERNACIONAL DE SOLUCIÓN ALTERNATIVA DE CONTROVERSIAS
}

\author{
Francisco ORREGO VICUÑA*
}

RESUMEN: El arbitraje como método alternativo de solución de controversias ha sido ampliamente debatido en los últimos tiempos, especialmente debido al desencadenamiento de controversias comerciales que la globalización ha traído consigo, razón por la cual es imprescindible prevenir dichas controversias y no sólo solucionar las que están en curso. En este sentido, el arbitraje constituye una alternativa viable que debe ser tomada en cuenta.

ABSTRACT: The arbitrage as an alternative controversy solution method has been widely discussed recently, especially due to the increasing number of commercial controversies the globalization has induced; reason why, it is necessary to prevent and not only solve the current controversies. In this sense, the arbitrage is a viable alternative that should be taken into account.

RÉSUMÉ: L'arbitrage c'est une méthode alternative de solution des controverses qui a eté discuté maintenant, spécialement grace à la plorifération des controverses commerciales que la mondialization a provoqué. C'est pour ça que c'est necessaire la prevention et solution de cettes controverses. L'arbitrage est une alternative plausible que doit être considerée.

* Profesor de derecho internacional en la Facultad de Derecho y en el Instituto de Estudios Internacionales de la Universidad de Chile. Miembro del Institut de Droit International. Preside el Tribunal Administrativo del Banco Mundial y es miembro de los Paneles de Árbitros y Conciliadores del Centro Internacional para ef Arreglo de Disputas en materia de Inversiones (CIADI). Juez ad-hoc en un caso ante el Tribunal Internacional del Derecho del Mar. 
SUMARIO: I. Nuevas respuestas para nuevas necesidades. II. ¿El arbitraje es parte de un sistema alternativo? III. Hay que mantener una distancia razonable de los tribunales. IV. Arbitraje de via rápida. V. Hay que perfeccionar los métodos tradicionales. VI. Nuevos enfoques alternativos. VII. Procedimientos para reclamos de monto menor. VIII. La intervención del Ombudsman y la auto-regulación. IX. La internacionalización de los métodos alternativos. X. El arbitraje y la solución alternativa: desarrollo de las interconexiones. XI. Hay que asegurar la confidencialidad. XII. Un marco común para el arbitraje y los métodos alternativos. XIII. Hay que alcanzar un papel global.

\section{NUEVAS RESPUESTAS PARA NUEVAS NECESIDADES}

En la medida que el comercio, las inversiones y los negocios se expanden a través del mundo a una velocidad creciente, los sistemas de solución de controversias se ven enfrentados a nuevos desafios. En efecto, tal como los procedimientos judiciales nacionales se han visto saturados por la demanda de sus servicios originada en las nuevas necesidades de economías en expansión y de la vida social, también los procedimientos internacionales de solución de controversias se ven enfrentados a la necesidad de encontrar respuestas adecuadas.

Un sistema internacional de solución alternativa de controversias, que combina los mecanismos tradicionales con nuevos enfoques, ya ha sido establecido y a no dudarlo será materia de importante perfeccionamiento en los años venideros.

Este artículo explorará algunas de las principales características de este nuevo sistema, con particular referencia al papel que en él desempeña el arbitraje.

\section{II. ¿EL ARBITRAJE ES PARTE DE UN SISTEMA ALTERNATIVO?}

Una primera pregunta que debe hacerse es si acaso el arbitraje forma en realidad parte de un nuevo sistema alternativo de solución de controversias. Históricamente el arbitraje se concibió como una alternativa a la solución de las controversias por los tribunales ordinarios de justicia, tanto en el plano nacional como en el ámbito del derecho internacional. 
¿Pero cuál es la situación hoy día, especialmente en lo que se refiere a comercio y negocios? En el plano nacional, al menos en los Estados Unidos de América y en el Reino Unido, el arbitraje es considerado en general como una forma de solución alternativa. En el plano internacional, sin embargo, la respuesta no es tan clara.

El sistema de Solución Amistosa de Controversias de la Cámara Internacional de Comercio (ICC-ADR), por ejemplo, excluye el arbitraje, aun cuando se examinan los reglamentos de la cámara en su conjunto se puede observar que descansan tanto en el arbitraje como en el sistema alternativo que se ha creado recientemente. ${ }^{1}$ Pero otras instituciones, como el London Court of International Arbitration (LCIA), no parecen compartir el mismo enfoque, y tanto el arbitraje como otros métodos se consideran como alternativas, cada una por cierto con sus propias características. $^{2}$

La respuesta probablemente se encuentre en el camino que el arbitraje probablemente recorra. ${ }^{3}$ Se ha observado que el arbitraje ha evolucionado hacia formas más estructuradas que hacen que sus diferencias con los procedimientos judiciales sean menos marcadas. ${ }^{4}$ En efecto, en muchas ocasiones la estrategia que siguen los abogados que litigan en un procedimiento arbitral no son diferentes de las que utilizan ante los tribunales ordinarios de justicia. Algunas veces también los árbitros tienden a enfocar el procedimiento de una manera similar.

Si este fuese el enfoque que predomine en el futuro, entonces es probable que los nuevos sistemas, caracterizados por su mayor flexibilidad y menor formalismo, se constituirán en una alternativa no sólo respecto de los tribunales ordinarios sino también respecto del propio arbitraje.

1 ICC, Guide to ICC ADR, junio de 2001.

2 LCIA, Dispute Resolution Services: Arbitration-Mediation.

3 Wang, Margaret, "Are Alternative Dispute Resolution Methods Superior to Litigation in Resolving Disputes in International Commerce?", Arbitration International, vol. 16, 2000, pp. 189-211. Veáse también Schwebel, Stephen M., International Arbitration: three salient problems, 1987; Hunter, Martin, "International Commercial Dispute Resolution: The Challenge of the Twenty-first Century", Arbitration International, vol. 16, 2000, pp. 379-392.

4 Gray, C. y Kingsbury, B., "Developments in Dispute Settlement: Inter-State Arbitration since 1945", British Year Book of International Law, 1992, pp. 97-134. 


\section{HAY QUE MANTENER UNA DISTANCIA RAZONABLE}

\section{DE LOS TRIBUNALES}

Hay todavía otra dimensión de esta materia que debe considerarse. La opción de una solución alternativa de controversias involucra en general un cierto grado de insatisfacción. Los objetivos de la solución alternativa se han descrito como la búsqueda de una solución "a la congestión de los tribunales, así como a sus costos y demoras, procurando incrementar la participación de la sociedad en el proceso de solución de controversias, facilitar el acceso a la justicia y proveer métodos más efectivos de solución de esas controversias". 5

La larga demora de los tribunales en resolver las controversias, la naturaleza costosa y compleja de los procedimientos y la falta de especialización para tratar de los nuevos problemas, han hecho que el recurso a los tribunales ordinarios sea muchas veces inaccesible o inefectivo. ${ }^{6}$ Los propios métodos alternativos no están desde luego exentos de críticas, ${ }^{7}$ pero en general son considerados como un enfoque viable para resolver esas dificultades.

El aspecto que debe destacarse es que el arbitraje mismo parece ser también una alternativa viable para solucionar esos mismos problemas, $y$ por tanto no es percibido con el mismo grado de insatisfacción que los tribunales ordinarios. La creciente demanda por el arbitraje y el papel activo que desempeñan las principales instituciones de arbitraje es indicativo de que no existe tal insatisfacción en lo que respecta a este método.

En este contexto puede entonces esperarse que si el arbitraje mantiene una distancia prudente de los procedimientos que caracterizan a los tribunales ordinarios continuará siendo parte de las soluciones alternativas como lo son otros métodos, salvaguardando siempre sus característi-

5 Applebey, George, "An Overview of Alternative Dispute Resolution", en Samson, Claude y McBride, Jeremy (eds.), Alternative Dispute Resolution, 1993, p. 27, con particular referencia a Goldberg et al, Dispute Resolution, 1985.

6 Ibidem, p. 30.

7 Baruch Bush, Robert A., "Dispute Resolution-The Domestic Arena: A Survey of Methods, Applications, and Critical Issues", en Vasquez, John A. et al. (eds.), Beyond Confrontation. Learning Conflict Resolution in the Post-Cold War Era, 1998, pp. 23-27. Veáse también Woodman, Gordon R., "The Altemative Law of Alternative Dispute Resolution", en Samson, Claude y McBride, Jeremy (eds.), op. cit, nota 5, pp. 580-605; Kriesberg, Louis, "Applications and Misapplications of Conflict Resolution Ideas to International Conflicts", en Vasquez et al., op. cit., pp. 87-102; y Vasquez, John A., "The Learning of Peace: Lessons from a Multidisciplinary Inquiry", en Vasquez et al., op. cit., en esta misma nota, pp. 211-238. 
cas particulares de asociación con el debido proceso y de permitir el método adversario en la solución de la controversia.

\section{ARBITRAJE DE VÍA RÁPIDA}

Existe, sin embargo, la necesidad de atender a los problemas de expedición. Si el arbitraje es demasiado lento en comparación a otros métodos alternativos podría crearse una desventaja para el primero.

Una tendencia reciente que es de interés considerar en este plano es la creación de vías rápidas para el desempeño del arbitraje con miras a alcanzar soluciones en un plazo corto, y disminuir los costos y otros problemas vinculados al arbitraje tradicional. Ya en 1978 la Cámara Internacional de Comercio estableció un Comité Permanente para la Reglamentación de las Relaciones Contractuales. Su objetivo era el de adoptar recomendaciones que las partes se comprometían a considerar de buena fe o bien adoptar decisiones que las partes se comprometían a respetar como si fuesen disposiciones contractuales, si bien no equivalían a una sentencia. ${ }^{8}$ La Cámara Internacional de Comercio también estableció en 1990 un procedimiento de referencia previa al arbitraje con el objetivo de solucionar los problemas causados por procedimientos arbitrales demasiado lentos. ${ }^{9}$

$\mathrm{Si}$ bien estas iniciativas no fueron enteramente exitosas, apuntaban a un aspecto del arbitraje internacional que debe tenerse en consideración. La Corte de Arbitraje de la Cámara Internacional de Comercio presta también atención permanente al cumplimiento de los plazos, recomendando que los procedimientos se completen en un periodo de seis meses. Arbitrajes de vía rápida también se han utilizado con éxito en aplicación de las reglas normales de arbitraje en la Cámara Internacional de Comercio cuando son aplicadas con un sentido de urgencia o expedición por el tribunal designado. ${ }^{10}$

Un mecanismo prometedor en este plano ha sido establecido por la Organización Mundial de Propiedad Intelectual mediante Reglas de Arbitraje Expedito, las que simplifican las Reglas de Arbitraje de la Organiza-

8 Paulsson, Jan, "Fast-Track Arbitration in Europe (with special reference to the WIPO Expedited Arbitration Rules)", Hastings International and Comparative Law Review, vol. 18, 1995, p. 714 .

9 ldem.

10 Ibidem, p. 717 
ción $^{11}$ para alcanzar resultados en forma más rápida. Los elementos claves de este mecanismo expedito son la designación de un solo árbitro y la simplificación de los plazos a lo largo del procedimiento.

\section{HAY QUE PERFECCIONAR LOS MÉTODOS TRADICIONALES}

Los métodos tradicionales de solución de controversias en el derecho internacional también han ido evolucionando rápidamente para adaptarse a las nuevas necesidades y demandas. Ello obedece primero al hecho de que los usuarios de los procedimientos internacionales de solución de controversias, como aquellos en el plano nacional, tienen una clara preferencia por resolver sus controversias mediante la negociación u otros métodos no confrontacionales, en tanto que la opción judicial se mantiene habitualmente como una última alternativa. ${ }^{12}$

Otro aspecto que estimula los arreglos alternativos es la necesidad de que los métodos contemporáneos de solución de controversias aseguren la prevención de las mismas, más que la solución de controversias ya en curso, objetivo que en muchas ocasiones sólo se puede alcanzar mediante la utilización de nuevos mecanismos. ${ }^{13}$ Como resultado de una ambiente que favorece la prevención, lo que además ayuda a reducir los riesgos jurídicos involucrados, ${ }^{14} \mathrm{y}$ de la disponibilidad de nuevos procedimientos, es dable esperar que habrá un creciente número de controversias sometidas a los procedimientos de prevención y resolución.

Como consecuencia de este desarrollo se pueden observar importantes perfeccionamientos en otros mecanismos de carácter tradicional: ${ }^{15}$

11 WIPO Arbitration Rules and Expedited Arbitration Rules, International Legal Materials, vol. 34,1995 , p. 559.

12 Stipanowich, Thomas J. y Kaskell, Peter H. (eds.), Commercial Arbitration at its Best, 2001, p. 5.

13 Shihata, Ibrahim F. I., "Implementation, enforcement and compliance with international environmental agreements: Practical suggestions in light of the World Bank's experience", Georgetown International Environmental Law Review, vol. 9, 1996, pp. 37-51.

14 Coombe, George W., "The Future: Implementing New Approaches to the Settlement of Transnational Commercial Disputes", Canada-United States Law Journal, vol. 17, 1991, p. 535.

15 Orrego Vicuña, Francisco y Pinto, Christopher, "Peaceful Settlement of Disputes. Prospects for the twenty-first Century (Report prepared for the Centennial of the First International Peace Conference)', en Kalshoven, F. (ed.), The Centennial of the First International Peace Conference, 2000, pp. 321-329. 
- Creciente utilización de las negociaciones y consultas, incluyendo requisitos de notificación, disponibilidad de información, monitoreo y mayor institucionalización.

- Investigación y aclaración de hechos de manera institucionalizada.

- Apoyo institucional a la conciliación y recurso a la conciliación obligatoria.

- Creciente papel de los órganos técnicos y de expertos en la determinación de las controversias.

- Desarrollo de la mediación.

\section{NUEVOS ENFOQUES ALTERNATIVOS}

Algunas experiencias nacionales tienen gran relevancia en función de estos otros enfoques alternativos. La solución alternativa de controversias ha sido el enfoque favorito en los Estados Unidos de América durante los últimos veinticinco años, así como en el Reino Unido y otros países más recientemente. ${ }^{16}$ Además de los métodos clásicos de negociación, conciliación, mediación y arbitraje, la solución alternativa de controversias incluye una variedad de otros métodos que se han desarrollado en el marco de estas experiencias nacionales.

Estas otras alternativas incluyen:

- Arbitraje ordenado por un tribunal ordinario.

- Arbitraje no obligatorio.

- Sentencia privada.

- Arriendo de un juez.

- Mediación combinada con arbitraje.

- Procedimiento sumario.

- Procedimiento sumario con un jurado.

- Evaluación inicial por un neutral.

- Investigación de hechos por un neutral.

- Diálogo de política. 
Además del interés que estos nuevos métodos tienen en sí, hay una experiencia importante en su aplicación a nuevas áreas. Algunas de estas áreas pueden tener una relevancia especial para la solución internacional de controversias.

\section{PROCEDIMIENTOS PARA RECLAMOS DE MONTO MENOR}

El establecimiento de procedimientos para reclamaciones de un monto menor, generalmente con relación a bienes de consumo, facilita de manera importante una justicia expedita y razonable en sus costos para aquellos reclamos que involucran montos pequeños. Esta experiencia tiene especial interés en su aplicación a contratos o transacciones internacionales. Esta opción no se encuentra generalmente disponible y los procedimientos normales son demasiado caros para el monto involucrado en la controversia o para los medios del reclamante..$^{17}$

La reciente experiencia de la solución de reclamaciones relativas a las cuentas secretas suizas, que estableció un procedimiento expedito y gratis para efectuar las reclamaciones, puede servir de ejemplo para tratar de las reclamaciones menores en general. ${ }^{18}$

Experiencias de interés también se encuentran en el caso de la Comisión de Indemnizaciones de Naciones Unidas y los mecanismos para la indemnización del trabajo esclavo. Procedimientos de vía rápida para reclamaciones menores también han sido desarrollados en algunas experiencias nacionales, particularmente la American Arbitration Association y el Judicial Arbitration and Mediation Services (JAMS). ${ }^{19}$

\section{LA INTERVENCIÓN DEL OMBUDSMANY LA AUTO-REGULACIÓN}

La experiencia británica en materia de intervención del Ombudsman es también de interés para la solución internacional de controversias. Esta experiencia incluye la Oficina del Ombudsman de Seguros, que provee desde consejos informales hasta arbitraje formal de controversias relacionadas con el negocio de los seguros; la Oficina del Ombudsman Banca-

17 Id., "Small Claims", op. cit., nota 5, pp. 45-51.

18 Buergenthal, Thomas, "Arbitrating Entitlement to Dormant Bank Accounts", en varios autores, Liber Amicorum Ibrahim F. I. Shihata, 2001, pp. 79-102.

19 Stipanowich y Kaskcll, op. cit., nota 12, at 58. 
rio; el Mecanismo del Ombudsman para Sociedades de Ahorro para la Construcción y el papel del árbitro de inversiones. ${ }^{20}$

En la medida en que estas actividades se relacionan crecientemente con la operación del mercado global, especialmente en lo que respecta a servicios y transacciones financieras, habrá necesidad de una alternativa que sea accesible al pequeño inversionista. El mismo éxito de la globalización en estas áreas requerirá de una alternativa de este tipo.

En algunas ocasiones, los mecanismos de auto-regulación de la industria han facilitado una alternativa, enfoque que debe promoverse, pero siempre existe la necesidad de contar con procedimientos de solución de controversias para el caso de que esos mecanismos no alcancen una solución razonable.

Nuevamente en el plano nacional algunos mecanismos de auto-regulación tienen importancia, como es el de las fusiones y adquisiciones en el Reino Unido, que prevén decisiones adoptadas por paneles con miras especialmente a la protección de los accionistas minoritarios, lo que no está exento de revisión judicial por algunas causas. ${ }^{21}$

Siendo las fusiones y adquisiciones tan comunes en una economía global, la participación de mecanismos alternativos de solución de controversias puede llegar a ser una característica principal del sistema internacional, especialmente para proteger a compañias pequeñas o accionistas minoritarios.

En la medida que la globalización avanza en los mercados comerciales y financieros, nuevos métodos alternativos y áreas para la solución de controversias se encontrarán crecientemente disponibles, complementando la labor de los tribunales y de los métodos tradicionales de solución de controversias. Estos desarrollos probablemente encontrarán modelos y experiencias relevantes en las experiencias nacionales que hoy se conocen.

20 Hodgin, R. W., "Ombudsmen and other Complaints Procedures in the Financial Services Sector in the United Kingdom", en Samson, Claude y McBride, Jeremy (eds.), op. cit., nota 5, pp. 168-194.

21 Tridimas, Takis, "Self-regulation and Investor Protection in the United Kingdom", en Samson, Claude y McBride, Jeremy (eds.), op. cit., nota 5, pp. 456-487. 


\title{
IX. LA INTERNACIONALIZACIÓN DE LOS MÉTODOS
}

\author{
ALTERNATIVOS
}

Puede apreciarse sin dificultad que algunos de los métodos mencionados no son desconocidos en materia de solución internacional de controversias, quizás de una manera menos estructurada y más como resultado de la práctica que de definiciones formales. La pregunta que debe tenerse presente es si acaso los métodos hoy disponibles en el plano nacional serán transplantados a la solución internacional de controversias para facilitar el logro de similares objetivos.

Debe observarse, primero, que la negociación, consulta, buenos oficios, investigación de hechos, conciliación y mediación son métodos bien conocidos en la solución tradicional de controversias internacionales. Algunas de sus actuales características fueron anteriormente señaladas.

La conciliación es otro método que ha sido desarrollado en cierta medida. Reglas de conciliación han sido aprobadas por el CIADI, Uncitral, la Cámara Internacional de Comercio -ahora fusionadas con los procedimientos de solución amistosa de controversias-, las Naciones Unidas y la Corte Permanente de Arbitraje. Debe observarse, sin embargo, que los usuarios han demostrado recientemente no favorecer este método de manera especial.

En segundo lugar debe apreciarse que algunas de las técnicas más relevantes están gradualmente apareciendo en la solución internacional de controversias. Un ejemplo es, por cierto, el procedimiento de solución amistosa de la Cámara Internacional de Comercio, que prevé no sólo la mediación sino también la evaluación por un neutral, un procedimiento sumario y otras técnicas de solución o una combinación de las mismas.

En tercer lugar también se está fortaleciendo la utilización internacional de expertos para la determinación de controversias. Tradicionalmente, esta alternativa se utilizaba en materia de investigación y establecimiento de los hechos, como también ha sido el caso de recurrir a la conciliación en conjunto con la investigación y establecimiento de los hechos. Esto último ocurre, por ejemplo, en las Reglas del CIADI. La utilización de expertos tiene hoy día, sin embargo, un carácter muy especializado, como ocurre, por ejemplo, con las Reglas de la Cámara Internacional de Comercio para el uso de Expertos en materia de Controversias sobre Crédito Documental o con el Centro Internacional de Expertos de la Cámara Internacional de Comercio. 
En cuarto lugar, la mediación se ha transformado en la alternativa más favorecida para la solución internacional de controversias. Numerosos centros nacionales ofrecen servicios de mediación, como es el caso del Centro para Recursos de Política (CRP), la American Arbitration Association y JAMS. En el plano internacional, la mediación también se encuentra fácilmente disponible, como es el caso del procedimiento de mediación de la Corte de Arbitraje Internacional de Londres o de los procedimientos de solución amistosa de la Cámara Internacional de Comercio. En este último caso, la mediación es el procedimiento que debe utilizarse en caso de que las partes no hayan escogido ninguna otra alternativa.

La mediación obligatoria y la mediación ordenada por un tribunal son también variaciones de este método. Algunos gobiernos, especialmente el británico, están exigiendo a las instituciones y servicios públicos recurrir a la mediación antes de intentar cualquier forma de acción judicial.

En quinto lugar, las reglas aplicables a la solución internacional de controversias han sido regularmente adaptadas para permitir la participación de nuevos usuarios o para atender a materias especializadas, como las controversias relativas al medio ambiente y a los recursos naturales.

\section{EL ARBITRAJE Y LA SOLUCIÓN ALTERNATIVA: DESARROLLO DE LAS INTERCONEXIONES}

Los desarrollos que se han explicado determinan que el arbitraje y otros métodos alternativos continuarán coexistiendo en la solución internacional de controversias. Varias conexiones han surgido entre el arbitraje y estos métodos, generalmente con miras a proveer alternativas adicionales a los usuarios. Esta mayor flexibilidad debe ser por cierto bienvenida.

Cabe notar en particular la disponibilidad actual de varios métodos híbridos, que incluyen:

- Arbitraje no obligatorio.

- Arbitraje con opciones de solución.

- Arbitraje con oferta final de las partes.

- Mediación seguida de arbitraje. 
Esta no es la ocasión para analizar las características de cada uno de estos híbridos, pero sólo para destacar que con frecuencia existe el recurso al arbitraje cuando estos métodos fracasan. Cuando estos métodos cuentan con una administración institucional, esta progresión de uno a otro se facilita enormemente.

\section{Separación entre el papel de mediador y de árbitro}

Un mediador puede ocasionalmente asistir a las partes en el proceso de preparación del arbitraje dentro de ciertas limitantes. El más importante de estos límites es el papel que el mediador mismo pueda desempeñar en el arbitraje. Como regla general, no se favorece que el mediador pueda servir ulteriormente de árbitro en la búsqueda de una solución a una determinada controversia (Reglas del CPR y Reglas de Mediación de la Asociación Nacional de Corredores de Acciones-NASD), como tampoco se favorece que un árbitro pueda posteriormente actuar como mediador en ese caso (AAA).

La razón de esta separación de papeles es la de facilitar las comunicaciones con el neutral, durante la mediación, y mejorar así las posibilidades de un arreglo, lo que no ocurriría si las pruebas o puntos de vista que se le presentan pueden ser posteriormente juzgados por la misma persona en un procedimiento contradictorio. Ello es particularmente delicado cuando la información se entrega de manera confidencial, pues puede tener además implicaciones para el debido proceso. ${ }^{22}$

En este contexto, la utilización del arbitraje con oferta final y la mediación seguida de arbitraje es un tanto limitada. ${ }^{23}$ En el primer método, también conocido como mediación con arbitraje de oferta final, la mediación es seguida por la presentación que cada parte hace de su oferta final al árbitro. La mediación seguida de arbitraje puede involucrar a diferentes neutrales, pero que normalmente se encuentran presentes o participan en ambas etapas del procedimiento seguido.

\section{Un papel complementario para la determinación por expertos}

La determinación hecha por expertos se interconecta en muchos aspectos con el arbitraje y debe ser en realidad considerada como comple- 
mentaria. En ocasiones la determinación de un experto se transforma en una sentencia o en un contrato obligatorio. ${ }^{24} \mathrm{~A}$ veces un experto integra el tribunal arbitral o lo asesora. Instituciones como el Dispute Review Boards o los Dispute Adjudication Boards involucran también un alto grado de intervención de expertos.

Una experiencia exitosa en materia de utilización de expertos se ha dado en el marco de algunos paneles de la Comisión de Indemnización de las Naciones Unidas. Como un resultado interesante de este enfoque se ha diseñado, por ejemplo, una metodología alternativa para la evaluación de daños, cuando en circunstancias de una reclamación la metodología normal demostró ser enteramente inapropiada. ${ }^{25}$

\section{Intercambio de procedimientos}

Otra conexión de importancia entre el arbitraje y los métodos alternativos se refiere a la conducción de procedimientos paralelos. En algunas normas institucionales no hay dificultad en que se desarrolle el arbitraje o la solución judicial mientras una mediación se encuentra en marcha (LCIA, NASD), aún cuando otros arreglos no favorecen este paralelismo (CPR). Desde luego que con el consentimiento de las partes, la mediación puede ser emprendida durante el desarrollo del arbitraje (CPR, AAA), dentro de los límites que se han explicado anteriormente acerca de la participación de un mismo individuo en las diferentes etapas.

\section{HAY QUE ASEGURAR LA CONFIDENCIALIDAD}

Algunas de las interconexiones examinadas plantean el problema general de la confidencialidad en el arbitraje y los procedimientos alternativos. Tradicionalmente el asegurar la confidencialidad ha sido un elemento esencial del éxito de estos mecanismos. Todos los principales sistemas de arbitraje internacional insisten en este punto. Las reglas y la práctica, en lo que se refiere a la publicación de sentencias, también exigen normalmente el asentimiento de las partes, o bien que se publiquen los nombres con el resguardo debido.

24 Ibidem, pp. 59-60.

25 United Nations Compensation Commission, First Report and Recommendations of the F/2 Panel, 1999. 
En la mediación y en los procedimientos alternativos, la confidencialidad es todavía más estricta, pues de lo contrario se llegaría a un fracaso seguro. Los procedimientos alternativos tanto nacionales como internacionales son explícitos en esta exigencia (LCIA, AAA, CPR, NASD, JAMS), hasta el punto de restringir la participación en las audiencias y de prohibir la transcripciones (LCIA).

La aplicación estricta de la confidencialidad es indispensable en los arbitrajes y otros procedimientos que se refieren normalmente a materias comerciales y de negocios. Recientemente, sin embargo, un punto de vista diferente se ha planteado en relación a la solución de controversias que se refiere al comercio y a las inversiones, pero insertadas en un contexto de mayor amplitud, que involucra materias de interés público. La confidencialidad en este otro contexto ha sido ocasionalmente criticada debido a que podría afectar la transparencia necesaria para asegurar que los intereses públicos más amplios se tomen en consideración. ${ }^{26}$

En respuesta a esta otra dimensión del arbitraje, $\operatorname{los}_{\text {paneles }}{ }^{27}$ y el órgano de apelación ${ }^{28}$ de la OMC han abierto sus procedimientos a la presentación de amicus curiae, por organizaciones que se interesan en los resultados de un caso. Si bien en términos generales estas presentaciones pueden contribuir a la mayor disponibilidad de información y puntos de vista de parte de las organizaciones apropiadas, hay también importantes problemas que deben resolverse.

Existe primero un problema respecto de los Estados miembros de la OMC que no son partes en la controversia, a los que no se les permite participar en los procedimientos. Se encuentran así en desventaja respecto de aquellas organizaciones que son autorizadas a participar. Pero el problema más serio es el de asegurar que sólo aquellas organizaciones de alta seriedad profesional y que tienen una trayectoria responsable sean autorizadas a participar.

Los mecanismos de solución de controversias del NAFTA están siguiendo un camino similar. Primero, mediante una orden del tribunal arbitral en el caso Methanex, instituido bajo las normas de Uncitral, se esta-

26 Public Citizen, Report on NAFTA Chapter // investor-to-State cases: Bankrupting Democracy, 2001.

27 WTO, United States-Import Prohibition of certain Shrimp and Shrimp Products, AB-1998-4, 12 de octubre de 1998, pfo. 13.

28 WTO Appellate Body Decision in the British Steel Case, AB-2000-1, WT/DS 138/AB/R, 10 de mayo de 2000, pfos. 36-42; y Decision in the Asbestos case, AB-2000-11, WT/DS 135/AB/R, 12 de marzo de 2001 , pfos. $50-57$. 
bleció que las presentaciones amicus curiae podían hacerse por escrito, sujetas a algunas limitaciones. ${ }^{29}$ La justificación que se invocó para esta decisión fue que la materia controvertida involucraba asuntos de interés público. ${ }^{30}$

Segundo, las notas interpretativas de algunas disposiciones del capítulo 11 del NAFTA, adoptadas por la Comisión de Libre Comercio, ${ }^{31}$ registran el acuerdo de las partes acerca de que no existe una obligación general de confidencialidad. Como consecuencia de ello se permite el acceso público a la documentación de un caso, con la exigencia de evitar el conocimiento de información comercial confidencial o de algunos otros tipos de información que deben protegerse.

Estos desarrollos plantean la interrogante siguiente: jen casos que involucran consideraciones de interés público, debería adoptarse una norma de confidencialidad diferente de la que se aplica a otros casos comerciales de tipo común? Cuando el caso es administrado institucionalmente, como ocurre en la OMC y el NAFTA, existe la posibilidad de que las normas aplicables sean clarificadas por los organismos pertinentes. $\mathrm{Si}$ ello no es así, entonces debe ser el tribunal quien decida sobre cualquier problema de interpretación de las reglas aplicables al arbitraje.

Hay todavía otra consecuencia de este nuevo enfoque en materia de confidencialidad. Si ese enfoque se generaliza, de modo probable el arbitraje se vería irremediablemente separado de los otros procedimientos alternativos y se asociaría más estrechamente a los procedimientos de los tribunales ordinarios de justicia. Dejaría así de ser una "alternativa" y sería solo una variable de la decisión judicial. Ello afectaría el papel del arbitraje y podría traducirse en un mayor interés por recurrir a los demás métodos alternativos. Puede entonces concluirse que la confidencialidad debe mantenerse como la regla general, sujeta únicamente a aquellas excepciones que se justifican a la luz de problemas $o$ arreglos especiales.

Tampoco debe perderse de vista que muchas de las entidades que postulan un enfoque diferente de la confidencialidad, tienen un interés específico en influenciar el resultado del proceso. Este interés no siempre es un interés público real. De ahí que también debe respetarse el principio fundamental de la independencia de los tribunales y de la necesidad de

29 Methanex $v$. United States, Order of the Tribunal, 15 de enero de 2001.

30 Ibidem, p. 13.

31 NAFTA Free Trade Commission, Notes of Interpretation of Certain Chapter 11 Provisions, 31 de julio de 2001 . 
abstenerse de influir directa o indirectamente en su trabajo. Árbitros de gran prestigio profesional estarán además siempre conscientes de los aspectos de interés público que puedan existir en los casos que le son sometidos.

\section{UN MARCO COMÚN PARA EL ARBITRAJE Y LOS MÉTODOS ALTERNATIVOS}

Puede entonces observarse que son muchas las interconexiones entre el arbitraje y los otros métodos alternativos, todas las cuales apuntan hacia un marco de complementación y no de antagonismo. La pregunta que debe formularse es si acaso estas conexiones serán aisladas y ocasionales o podrán llegar a constituir un marco común.

\section{Incremento de los auspicios institucionales}

Debe apreciarse primero que el arbitraje y los métodos alternativos se están integrando de manera efectiva en el marco institucional común de los grandes sistemas de solución de controversias. Esta tendencia habrá por cierto de continuar. Podría incluso pensarse que no sería conveniente que en la Cámara Internacional de Comercio pueda llegar a disociarse el arbitraje de la solución alternativa como consecuencia de la existencia de reglamentaciones separadas, como también podría ser de interés para el CIADI el desarrollar un sistema de métodos alternativos más amplio, paralelo al arbitraje y a la conciliación existente, quizás en el marco de un acuerdo opcional.

\section{Un nuevo manejo de los conflictos corporativos}

En segundo lugar, puede apreciarse que las empresas están crecientemente recurriendo a un "manejo de las controversias", incluyendo en dicho esquema no sólo el arbitraje en forma aislada, sino que una cadena de métodos que van de la negociación a la mediación y luego al arbitraje, cada uno con sus propias características y a su debido tiempo. ${ }^{32}$

Una razón poderosa para seguir este enfoque es que las técnicas de solución alternativa ofrecen la posibilidad de tener en consideración el interés continuo de las partes en salvaguardar sus relaciones mutuas y no

32 Stipanowich y Kaskell, op. cit., nota 12, pp. 6-9. 
solamente en solucionar una controversia que las ha dividido como puede ocurrir en el arbitraje. ${ }^{33}$ Esta relación de continuidad es esencial para la realización de los negocios en un ambiente global. Por cierto, el arbitraje estará siempre disponible como el método de última instancia.

\section{Un sistema estructurado de solución internacional de controversias}

En tercer lugar, resulta también claro que la comunidad internacional se dirige hacia el establecimiento de un sistema estructurado de solución internacional de controversias en el que interactuarán tanto los tribunales públicos como los mecanismos privados. ${ }^{34}$ Todos estos elementos forman naturalmente parte de un sistema general que les es común. Como tales deben verse crecientemente interrelacionados y no aislados como muchas veces ocurre hoy a consecuencia de la fragmentación del derecho y de los procedimientos de solución de controversias.

No todos los mecanismos deben por cierto integrarse bajo una estructura común. Tampoco ello debiera conducir a una jerarquía estricta de tribunales internacionales o métodos, pues ello afectaría la libertad de opción que tienen las partes o la flexibilidad necesaria para acordar formas específicas de solución de controversias en el contexto de determinadas actividades o problemas. Es más bien un esfuerzo por llevar a la atención de las partes, de una manera organizada, las variadas opciones que tienen a su disposición, y como podrían obtener ventaja de estas alternativas.

Las experiencias nacionales proveen algunos criterios acerca de la posible interconexión de los tribunales y los métodos alternativos. El caso típico es, por ejemplo, el de un arbitraje ordenado por un tribunal o cuando los tribunales ordenan a las partes proseguir alguna alternativa determinada. Esta misma posibilidad existe en los acuerdos de arbitraje que permiten considerar una mediación y otros métodos alternativos (AAA, CPR). La Corte Internacional de Justicia ha dirigido a las partes a explorar acuerdos mediante la negociación en más de una oportunidad. ${ }^{35}$

33 Ibidem, p. 13.

34 Orrego Vicuña, Francisco, Dispute Settlement for a New Century, Sir Hersch Lauterpacht Memorial Lectures 2001, a ser publicado por Cambridge University Press.

35 Permanent Court of International Justice, Free Zones Case, Ser. A., núm. 24, 1929, p. 12. En el caso Gabcikovo-Nagymaros, la Corte Internacional de Justicia guió a las partes acerca de algunos problemas juridicos y las partes se comprometieron a emprender negociaciones bilaterales sobre estas bases; Report of the International Court of Justice by President Stephen M. Schwebel to the United 
Nuevamente, aquí se hace necesario introducir una anotación cautelosa. Varias proposiciones se han hecho recientemente para establecer una Corte Internacional de Apelaciones de Arbitraje o una Corte Internacional de Sentencias Arbitrales. ${ }^{36}$ También se ha propuesto transformar el sistema de solución de controversias de la OMC en una Corte de Comercio Internacional.

Todas estas iniciativas tienen por cierto un gran interés. El problema radica en que eventualmente podrían conducir al arbitraje a una situación muy similar a la de la solución judicial y, por tanto, sería menos una "alternativa". Por las razones que se han indicado, este resultado debe en lo posible evitarse.

\section{HAY QUE ALCANZAR UN PAPEL GLOBAL}

El arbitraje y los métodos alternativos tienen un futuro en común que debe ser preservado. En la medida que estos métodos sean organizados, orientados y promovidos, el sistema alternativo de solución internacional de controversias que ha venido emergiendo gradualmente se transformará en los años venideros en una auténtica realización.

Cada método en sí mismo ha venido evolucionando para satisfacer nuevas necesidades y realidades, y nuevos métodos se encuentran disponibles. Las instituciones públicas y privadas están proveyendo mecanismos globales muy valiosos en algunos campos. La administración de la justicia internacional ampliamente concebida está integrándose de manera generalizada con un sistema de métodos alternativos que le son complementarios, lo que en realidad es la única manera de hacer justicia respecto de las demandas crecientes de la comunidad internacional.

Nations General Assembly: Fifty-second Session, 36th Plenary Meeting, 27 de octubre de 1997, Doc. A/52/PV. 36, pp. 2 y 3.

36 Holzmann, H., "A Task for the 21st Century: Creating a New International Court for Resolving Disputes on the Enforceability of Arbitral Awards", en Hunter, M. et al., The Internationalization of International Arbitration, 1995, pp. 109-114; Schwebel, S. M., "The Creation and Operation of an International Court of Arbitral Awards", op. cit., en esta misma nota , at 115-123. 